Supporting Information

\title{
Elimination of stacking faults in semipolar GaN and light emitting diodes grown on sapphire
}

Jie Song ${ }^{1,2 *}$, Joowon Choi ${ }^{1,2}$, Cheng Zhang ${ }^{1}$, Zhen Deng ${ }^{1}$, Yujun Xie ${ }^{3}$, and Jung Han ${ }^{1}$

${ }^{1}$ Department of Electrical Engineering, Yale University, New Haven, CT 06520, USA

${ }^{2}$ Saphlux Inc, Branford, CT 06405, USA

${ }^{3}$ Department of Mechanical Engineering \& Materials Science, Yale University, New Haven, CT 06520, USA

*Email: jiesong132@gmail.com 


\section{Schematic drawing of $\mathrm{OCE}$}

Figure S1 shows the schematic drawing about process of orientation control epitaxy OCE for semi/non-polar GaN grown on PSS or Si substrates. (a), Choose the right sapphire or Si substrates. (b), Pattern the substrate into stripe trenches with one sidewall nearly parallel to sapphire c-plane or $\mathrm{Si}(111)$ plane. (c), Deposit dielectric mask on the surface of patterned substrate, except the sidewalls which are parallel to sapphire c-plane or $\operatorname{Si}(111)$ plane. (d), Selectively grow GaN on exposed sidewalls of patterned substrate. (e), Continue to grow GaN and coalese the stipes to form a thick continues semipolar GaN film. The normal surface direction will be GaN semipolar direction. 


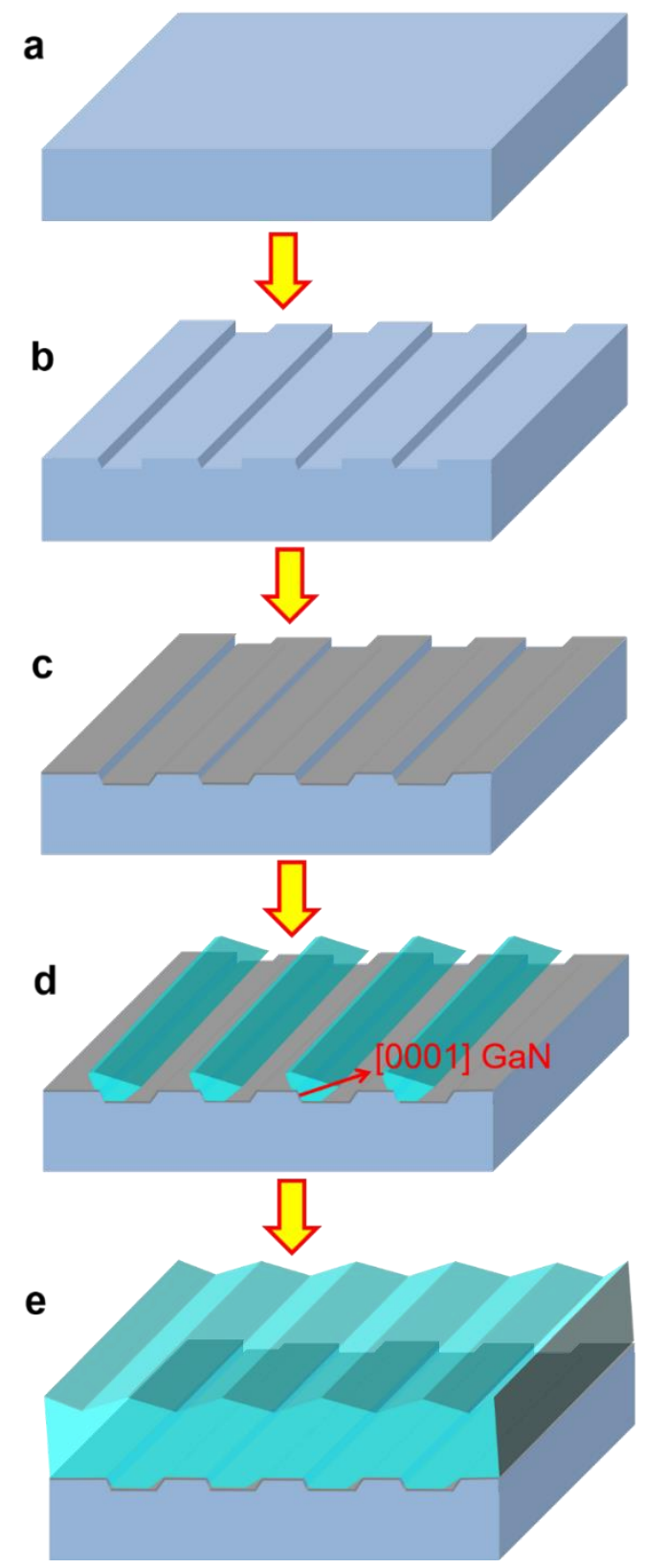

Figure S1. Schematic drawing about semipolar GaN grown on patterned sapphire substrates. (a) Choose the right sapphire or Si substrates. (b) Pattern the substrate into stripe trenches with one sidewall nearly parallel to sapphire c-plane or $\operatorname{Si}(111)$ plane. (c) Deposit dielectric mask on the surface of patterned substrate, except the sidewalls which are parallel to sapphire c-plane or Si(111) plane. (d) Selectively grow $\mathrm{GaN}$ on exposed sidewalls of patterned substrate. (e) Continue to grow $\mathrm{GaN}$ and coalese the stipes to form a thick continues semipolar GaN film. The normal surface direction will be GaN semipolar direction. 


\section{Study of SAG and kinetic Wulff plot}

The selective area growth (SAG) was conducted on $\mathrm{SiO}_{2}$-masked openings of annular ring and circle pattern on a-plane GaN/sapphire templates, as shown in Figure S2A and S2B. After growth, GaN crystal with different planes appears and the crystal planes can be identified and labeled in SEM image, as shown in Figure S3C and S3D. Figure S3C and S3D show the topview and $45^{\circ}$-tilted-view SEM images of GaN SAG on an annular ring pattern, respectively.

a

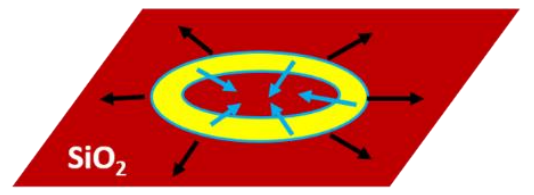

C

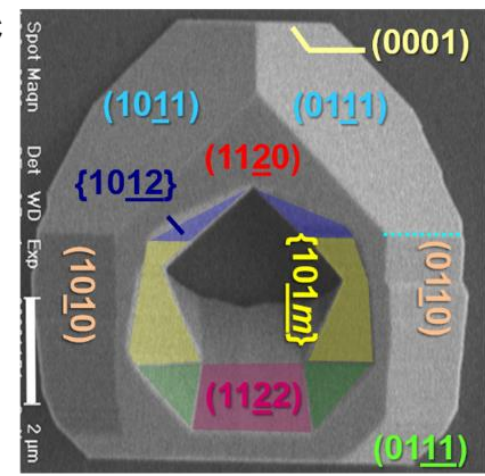

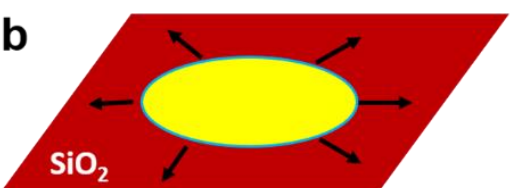

d

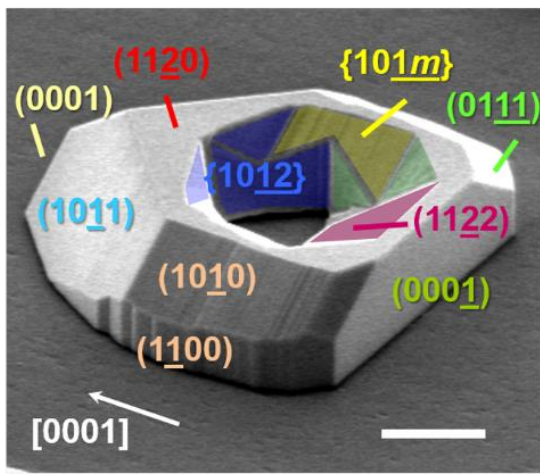

Figure S2. Schematic drawing of (a) annular ring and (b) circle pattern, respectively. (c) Top-view and (d) $45^{\circ}$-tilted-view SEM images of GaN SAG on an annular ring pattern. The scale bars are $2 \mu \mathrm{m}$ in both (c) and (d).

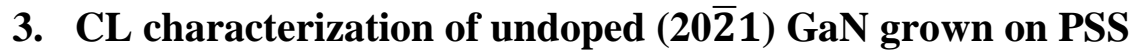

Figure S3 shows a panchromatic CL image of an undoped (20리) GaN grown on PSS. The dark band observed on the image are caused by the concentrated basal-plane stacking faults. The dark spots are corresponding to the threading dislocations. 


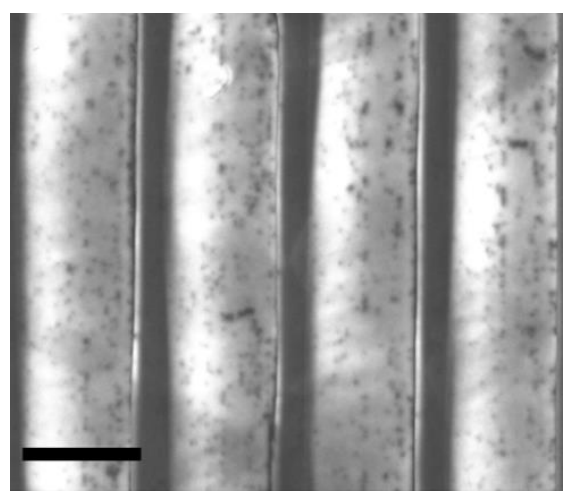

Figure S3. Panchromatic CL image of an undoped (202̄1) GaN grown on PSS. Scale bar is $5 \mu \mathrm{m}$. 